Revue d'histoire de l'Amérique française

REVUE D.HISTOIRE DE L'AMÉRIQUE FRANÇAISE

\title{
Les moulins de Terrebonne (1720-1775) ou les hauts et les bas d'une entreprise seigneuriale
}

\section{Solange De Blois}

Volume 51, numéro 1, été 1997

URI : https://id.erudit.org/iderudit/305622ar

DOI : https://doi.org/10.7202/305622ar

Aller au sommaire du numéro

Éditeur(s)

Institut d'histoire de l'Amérique française

ISSN

0035-2357 (imprimé)

1492-1383 (numérique)

Découvrir la revue

Citer cet article

De Blois, S. (1997). Les moulins de Terrebonne (1720-1775) ou les hauts et les bas d'une entreprise seigneuriale. Revue d'histoire de l'Amérique française, 51(1), 39-70. https://doi.org/10.7202/305622ar
Résumé de l'article

Cet article aborde un thème jusqu'ici négligé par les historiens de la Nouvelle-France : celui de l'industrie et de son destin au dix-huitième siècle. Terrebonne est, dès 1721, dotée de puissants moulins produisant farines et bois pour l'exportation et le marché intérieur. L'entreprise connaît un succès inégal au long de la période observée. L'administration de l'abbé Louis Lepage s'étend de 1720 à 1744 et s'achève par la faillite du propriétaire de Terrebonne. L'arrivée du nouveau seigneur, Louis de La Corne, coïncide avec le début de la guerre de Succession d'Autriche. La situation est évidemment plus favorable aux entrepreneurs. Mais la réussite des La Corne dépend aussi d'autres facteurs. À l'encontre de Louis Lepage, ils ont d'autres sources de revenus que Terrebonne et leurs entreprises industrielles s'appuient sur une solide fortune foncière. De plus, ils font partie d'un réseau complexe et étendu d'alliances. 


\title{
LES MOULINS DE TERREBONNE (1720-1775) OU LES HAUTS ET LES BAS D'UNE ENTREPRISE SEIGNEURIALE ${ }^{1}$
}

\author{
SOLANGE DE BLOIS \\ Département d'histoire \\ Université McGill
}

\section{RÉSUMÉ}

Cet article aborde un thème jusqu'ici négligé par les historiens de la NouvelleFrance: celui de l'industrie et de son destin au dix-huitième siècle. Terrebonne est, dès 1721 , dotée de puissants moulins produisant farines et bois pour l'exportation et le marché intérieur. L'entreprise connaît un succès inégal au long de la période observée. L'administration de l'abbé Louis Lepage s'étend de 1720 à 1744 et s'achève par la faillite du propriétaire de Terrebonne. L'arrivée du nouveau seigneur, Louis de La Corne, coïncide avec le début de la guerre de Succession d'Autriche. La situation est évidemment plus favorable aux entrepreneurs. Mais la réussite des La Corne dépend aussi d'autres facteurs. À l'encontre de Louis Lepage, ils ont d'autres sources de revenus que Terrebonne et leurs entreprises industrielles s'appuient sur une solide fortune foncière. De plus, ils font partie d'un réseau complexe et étendu d'alliances.

\section{ABSTRACT}

This article addresses a topic hitherto neglected by historians of New-France: the possibilities and limits of industry in the eighteenth century. As early as 1721, Terrebonne was equipped with grist and saw mills producing flour and timber for the export and internal trade. The entreprise's performance was uneven over the observed period. Abbé Louis Lepage's administration, which ran from 1720 to 1744, ended in bankrupcy. Terrebonne's purchase by a new seigneur, Louis de La Corne, coincided with the onset of the War of Austrian Succession. This new context offered better business opportunities, yet the La Cornes' success was also dependent on other factors. Unlike Louis Lepage, they had other sources of income, possessed valuable real estate to support their industries, and were part of a large and complex alliance network.

1. Cet article est tiré de notre mémoire de maîtrise: Possibilités et limites d'une entreprise seigneuriale: les moulins de Terrebonne, 1720-1775, mémoire de M.A. (Histoire), Université de Montréal, 1995. Nous remercions Peter Cook, Catherine Desbarats et Thomas Wien qui ont lu et commenté une version antérieure de notre texte. Nous remercions également les évaluateurs anonymes de la revue. 
Il existe plusieurs monographies consacrées à l'histoire d'une seigneurie sous le Régime français ${ }^{2}$. Toutefois, la seigneurie de Terrebonne, dotée d'une armature industrielle (minoterie et scierie ${ }^{3}$ ) dès le début des années 1720 , suit une voie de développement distincte, à certains égards, de celle des seigneuries étudiées jusqu'ici par les historiens de la Nouvelle-France. La colonisation y progresse lentement et par à-coups, comme c'est le cas de bien d'autres territoires ${ }^{4}$, particulièrement ceux qui se trouvent en périphérie. Mais, et c'est là l'un de ses traits originaux, alors que le nombre des terres occupées en zone agricole stagne, son village connaît une croissance précoce et rapide. Cet apparent paradoxe s'explique lorsqu'on campe Terrebonne dans l'ensemble régional constitué par les seigneuries de la rive nord de Montréal, en plein essor à l'époque qui nous occupe: l'île-Jésus, Terrebonne, Lachenaie, Repentigny, Saint-Sulpice et, à un moindre degré, Lavaltrie; et qu'on lie le phénomène à la présence sur les lieux des moulins à farine et à scie les plus imposants de la colonie (en dimension et en capacité, sinon pour le volume de leur activité). Car il n'y a pas de doute, c'est la minoterie, ou moulin marchand ${ }^{5}$, une immense bâtisse de pierre à 3 étages et 4 moulanges, assise sur la rivière des Mille-Îles, qui, drainant une partie des surplus agricoles de toute la région, génère l'activité indispensable à la croissance du bourg.

2. Louise Dechêne, Habitants et marchands de Montréal au XVII siècle (Montréal, Boréal, 1988) (édition originale, Montréal et Paris, Plon, 1974); Sylvie Dépatie, L'évolution d'une société rurale: l'île Jésus au XVIII siècle, thèse de Ph.D., Université McGill, 1988; Thomas Wien, Peasant Accumulation in a Context of Colonization, Rivière-du-Sud, Canada, 1720-1775, thèse de Ph.D., Université McGill, 1988; Louis Lavallée, La Prairie en NouvelleFrance, 1647-1760. Étude d'histoire sociale (Montréal, McGill-Queen's University Press, 1992); voir aussi Mario Lalancette, «La seigneurie de l'île-aux-Coudres au XVIII' siècle», dans Sylvie Dépatie et al., Contributions à l'étude du Régime seigneurial canadien (Montréal, Hurtubise HMH, 1987), 85-152; et Philippe Jarnoux, «La colonisation de la seigneurie de Batiscan aux XVII ${ }^{e}$ et $\mathrm{XVIII}^{e}$ siècles: l'espace et les hommes», Revue d'histoire de l'Amérique française, 40,2 (automne 1986): 163-191.

3. Auxquelles s'adjoindront en 1738 les bâtiments et l'équipement nécessaires à l'implantation d'une forge. À noter que la minoterie, qui est un puissant moulin à farine doté d'un crible mécanique actionné par l'eau et le mouvement d'une des meules, est à la fois moulin banal, puisqu'il dessert les censitaires, et entreprise industrielle.

4. Louis Michel, «L'économie et la société rurale dans la vallée du Saint-Laurent aux $\mathrm{XVII}^{e}$ et XVIII ${ }^{e}$ siècles: bilan historiographique», communication présentée au colloque Vingt ans après "Habitants et marchands de Montréal», la recherche sur les XVII' et XVIII" siècles canadiens, Montréal, 13 et 14 mai 1994.

5. Les moulins marchands se distinguent des moulins tout bonnement banals, à vent ou à eau; ces derniers, le plus souvent en pièce sur pièce, sont pour la plupart «au-dessous de tout», nous dit Louise Dechêne. Ils sont faits de pierre, comportent deux ou trois étages et logent des greniers. Ils contiennent en général deux ou trois moulanges, sont élevés sur un fort courant et une digue en maçonnerie maintient leur niveau d'eau assez haut pour que les roues puissent tourner à l'année. Louise Dechêne, Le partage des subsistances au Canada sous le Régime français (Montréal, Boréal, 1994), 33 et 35. 
L'étude des sources d'approvisionnement du moulin marchand et des marchés potentiels pour le bois et les farines nous apprend en effet qu'il faut déborder de la sphère locale de production et d'échange pour comprendre l'évolution économique de Terrebonne.

Il faut de plus tenir compte de l'action des seigneurs qui a été déterminante dans le développement économique de la seigneurie. Car si celle-ci est un pouvoir qui s'interprète, comme le remarque Louise Dechêne, c'est aussi une affaire qui se gère.

L'historiographie du Régime français a successivement présenté le seigneur comme un entrepreneur en peuplement encadré par l'État; comme un absent; et comme un gestionnaire minutieux profitant des privilèges que lui accorde le régime seigneurial pour extraire des revenus à même la production de ses tenanciers sans investir, sinon minimalement, ni participer autrement au développement économique local $^{6}$. Ce ne serait que vers la fin du XVIII ${ }^{\mathrm{e}}$ siècle, après le Régime français, que serait apparu, sous les traits de Gabriel Christie (et la plume de Françoise Noël), le type du «seigneur entrepreneur ${ }^{7} »$. Or, à Terrebonne, l'abbé Lepage qui achète la seigneurie en $1720^{8} \mathrm{y}$ injecte aussitôt d'importants capitaux, construit des moulins et s'engage à fond dans la gestion de son entreprise ${ }^{9}$. Ce qui n'est pas une garantie de succès, on le verra.

6. Marcel Trudel, Le régime seigneurial (Ottawa, Société historique du Canada, coll. «Brochure de la SHC», $\mathrm{n}^{\circ} 6$, 1967), 15; Guy Frégault, La civilisation de la Nouvelle-France, 1713-1744 (Ottawa, Fides, 1969), 148-150; Fernand Ouellet, Histoire économique et sociale du Québec, 1760-1850, 1 (1971): 9; Louise Dechêne, «L'évolution du régime seigneurial au Canada. Le cas de Montréal aux XVII ${ }^{e}$ et XVIII ${ }^{e}$ siècles», Recherches sociographiques, 12 (maiaoût 1971): 143-183; Sylvie Dépatie et al., Contributions... (Montréal, 1987); Allan Greer, Peasant, Lord and Merchant. Rural Society in Three Quebec Parishes, 1740-1840 (Toronto, 1985).

7. Françoise Noël, The Christie Seigneuries Estate Management and Settlement in the Upper Richelieu Valley, 1760-1859 (Montréal, 1992). L'expression est de Jean-Claude Robert qui désigne ainsi un seigneur du XIX ${ }^{\mathrm{e}}$ siècle: Jean-Claude Robert, «Un seigneur entrepreneur, Barthélémy Joliette, et la fondation du village d'Industrie (Joliette), 1822-1850», Revue d'histoire de l'Amérique française, 26,3 (décembre 1972): 375-395. Joseph-Noël Fauteux, qui écrit en 1927, nous présente de nombreux seigneurs ayant investi temps et argent dans l'industrie sous le Régime français. Toutefois il les décrit, suivant la rhétorique de la correspondance officielle et des adresses au roi, non comme des gens d'affaires recherchant leurs profits, mais des hommes de mérite se dévouant pour le bien public. Joseph-Noël Fauteux, Essai sur l'industrie au Canada sous le Régime français (Québec, 1927), 2 vol.

8. À l'abbé Louis Lepage succède, en 1744, Louis de La Corne dit l'aîné, écuyer et capitaine d'un détachement des Troupes de la Marine. Au décès de ce dernier, en 1762, sa femme, Élisabeth de Ramezay, prend en main l'administration de la seigneurie au nom de ses enfants mineurs. En 1772, Louis, le fils aîné, devient à son tour seigneur en titre.

9. Il n'est d'ailleurs pas le seul seigneur en ce début du XVIII ${ }^{e}$ siècle à se mêler activement du développement économique local. Ils sont peu nombreux, il est vrai, ceux qui possèdent le capital nécessaire pour se lancer en affaires, mais des membres de la classe marchande et de la noblesse militaire, enrichis souvent dans le commerce des fourrures, construisent ou 
Abordant l'étude de l'entreprise seigneuriale, nous nous sommes en effet heurtée à la question des raisons du succès ou de l'insuccès, selon le cas, des seigneurs de Terrebonne. Si la réussite et son contraire relèvent des limites structurelles de l'économie et des fluctuations de la conjoncture, ils dépendent aussi pour beaucoup des choix et des décisions des acteurs sociaux - en l'occurrence les seigneurs. Il faut ajouter que les moyens d'action des entrepreneurs sont tributaires de leur condition sociale et, encore davantage, de leur insertion, celle-ci facilitée par celle-là, dans les réseaux économiques et politiques à l'échelle régionale et coloniale. L'origine du capital investi par exemple, et cela a des répercussions sur les modes d'exploitation des moulins aussi bien que la conjoncture, diffère d'un seigneur à l'autre. Louis Lepage provient d'un tout autre milieu que Louis de La Corne qui achète la seigneurie en 1744. Ambitieux et très entreprenant, il a une manière bien à lui d'administrer ses affaires et elle se démarque totalement de celle de La Corne et de ses héritiers. La faillite du premier et le succès (relatif?) des seconds ne s'expliquent que si l'on tient compte de tous ces facteurs.

Notre propos n'est pas de retracer les étapes de la colonisation de Terrebonne ou l'histoire de son village ${ }^{10}$. Nous traiterons brièvement de ces questions, en quelques pages qui font le point à partir des données présentées dans notre mémoire de maîtrise. Le présent article a pour sujet l'entreprise seigneuriale et met l'accent sur l'analyse de la réussite ou de l'échec des entrepreneurs qui se succèdent à sa tête entre 1720 et 1775 .

Nous allons, en premier lieu et rapidement, présenter le cadre physique de l'enquête: territoire, population et habitat. Nous passerons ensuite à l'étude de l'entreprise - faisant leur part aux moulins à farine et à scie, mais aussi à la tentative d'implantation d'une forge par l'abbé Lepage - et de ses modes d'intégration aux circuits d'échanges qui la font vivre. Car ce qu'il nous faut établir, c'est comment et à quel point les moulins étaient articulés à l'économie globale puisque c'est la force des liens existant entre l'industrie et les marchés qui conditionne, au premier chef, sa survie.

exploitent des minoteries et des scieries, voulant profiter de la croissance de la production agricole et de l'expansion du commerce des farines et du bois. Voir, pour ce qui est des céréales, Louise Dechêne, «La mise en marché», Le partage..., 69-96; et, pour les scieries, Joseph-Noël Fauteux, Essai sur l'industrie...,vol. 1, étude générale qui demeure la plus complète sur ce secteur de l'activité industrielle en Nouvelle-France.

10. Pour cela nous renvoyons le lecteur aux chapitres deux et trois de notre mémoire de maîtrise. 


\section{LA SCÈNE}

\section{Le territoire}

Située sur la rive nord de la rivière des Mille-Îles et mesurant à l'origine deux lieues sur deux lieues, la seigneurie de Terrebonne fait face à l'extrémité nord-est de l'île Jésus et est bornée à l'est par Lachenaie et à l'ouest par la seigneurie des Mille-îles (figure 1). Ses premiers habitants s'y sont installés au tournant du XVIII e siècle, débordant vraisemblablement de Lachenaie où le front de la rivière est entièrement occupé dès $1699^{11}$. Ils s'aligneront d'est en ouest à partir des limites de Lachenaie, sur la côte de Terrebonne, leurs habitations faisant face à la rivière.

La seigneurie est traversée presque de part en part par la rivière Mascouche qui prend sa source dans la seigneurie de Blainville et se déverse dans la rivière des Mille-̂̂les, après un long détour par Lachenaie, près de la ligne qui sépare cette dernière de Terrebonne. La Mascouche se partage en deux tronçons juste avant de se perdre dans les terres non concédées qui limitent Terrebonne dans sa profondeur. Le ruisseau Saint-Pierre ou Bras Sainte-Marie (qui plus tard se nommera ruisseau La Corne) sillonne un territoire qui deviendra partie intégrante de la seigneurie de Terrebonne: l'augmentation des Plaines, octroyée en 1731 à l'abbé Lepage par l'intendant.

Une deuxième prolongation qui, comme la première, mesure deux lieues de front sur deux lieues de profondeur, sera concédée en 1752 à Louis de La Corne, par l'intendant Bigot, triplant la superficie initiale du territoire occupé par la seigneurie de Terrebonne.

À l'époque de l'abbé Lepage, la seigneurie était composée: 1) de la mouvance; 2) de huit arrière-fiefs, tous dans les Plaines; 3) de six domaines réservés par le seigneur dont quatre ont leur devanture sur la rivière des Mille-îles ${ }^{12}$ et deux se trouvent dans l'augmentation des Plaines. Toutes ces réserves, à l'exception de la première, située près de la ligne de partage entre Terrebonne et Lachenaie, et de la troisième où se trouvent le village, l'église et les moulins, logent une ferme exploitée le plus souvent en métairie ${ }^{13}$. Les moulins à farine et à scie,

11. Mario Nadon, Le premier demi-siècle de Lachenaie (1670-1724), mémoire de M.A. (Histoire), Université de Montréal, 1983, 38.

12. Le plus important parmi ceux-ci, celui de la Pointe aux Pins, fait 17 arpents de front et se situe, en partant de Lachenaie, aux deux tiers de la distance qui la sépare de la seigneurie des Mille-îles.

13. Aveu et dénombrement de Terrebonne, 20 mai 1736. Aveux et dénombrements du Régime français, vol. 2, f. 430v. 


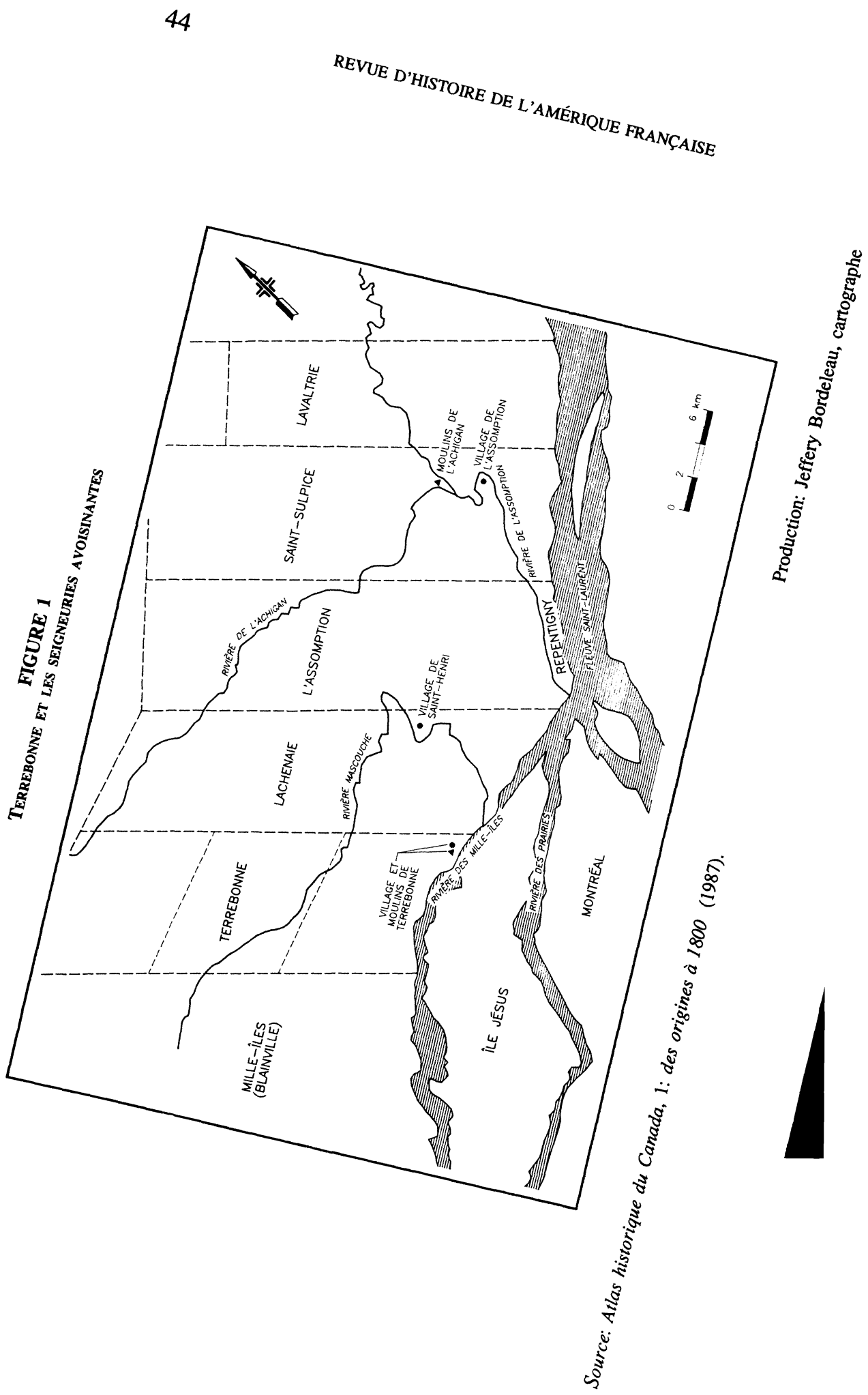


auxquels sont adjoints des hangars, sont situés sur une île qu'une digue en pierre relie au village.

Le contrat de vente de la seigneurie à Louis de La Corne, rédigé en janvier 1745, ne signale plus que le domaine de la Pointe aux Pins. Sans doute les autres ont-ils été peu à peu divisés et donnés en concession. Quant aux arrière-fiefs, ils seront réunis au domaine en 1749 par ordonnance de l'intendant, faute d'avoir été mis en valeur.

\section{La population et l'habitat}

La marche du peuplement piétine à Terrebonne, tout au long de la période étudiée et même au-delà (tableau 1). Mise à part Lavaltrie, Terrebonne compte la plus faible population parmi les seigneuries de la région et cela, jusqu'aux années 1760 .

Différentes raisons expliquent la situation. Terrebonne fait figure de seigneurie périphérique et reste en marge des mouvements de population qui contribuent à la colonisation des paroisses situées au nord de l'île de Montréal. L'Île-Jésus lui fait écran (jusqu'à ce qu'ellemême soit remplie ${ }^{14}$ ), d'une part, et, d'autre part, les fils des paysans de Lachenaie et de Repentigny migrent plus volontiers vers l'est que vers l'ouest ${ }^{15}$. De plus, Terrebonne ne bénéficie pas comme l'île-Jésus et Saint-Sulpice de la présence de seigneurs solidement établis (toutes deux sont des seigneuries ecclésiastiques appartenant respectivement au séminaire de Québec et aux Sulpiciens) et capables d'orienter et d'encourager la colonisation. L'abbé Lepage est un individu isolé en comparaison et, pendant longtemps Louis de La Corne, son successeur, pris par ses obligations militaires et n'habitant pas sur les lieux, n'octroiera que très peu de terres: 17 entre 1745 et $1757^{16}$.

Un autre facteur entrave le mouvement d'occupation du sol sur ce territoire: dans la seconde moitié du siècle, les terres disponibles dans la zone agricole sont situées aux confins des Plaines ou dans la continuation $\mathrm{La}$ Corne et se trouvent donc très éloignées des services, église et moulin, et peu ou pas irriguées.

Le caractère périphérique de la seigneurie de Terrebonne est encore plus marqué en fin de période. Entre 1765 et 1790 (comme le montre le tableau 2), alors que les autres seigneuries de la région présentent des taux de croissance de population très élevés par rapport

14. Et même alors, l'île ne se déverse pas seulement dans Terrebonne, cela va de soi. Ainsi, Blainville sera le réceptacle naturel des excédents de Sainte-Rose. Solange De Blois, Possibilités et limites..., 47.

15. Ibid., 21-22.

16. Voir le graphique II dans l'annexe B de notre mémoire de maîtrise. 


\section{TABLEAU 1}

Progression de la population régionale

\section{1-1790}

Seigneuries

Années

\begin{tabular}{|c|c|c|c|c|c|c|c|c|}
\hline & 1721 & 1730 & 1732 & 1736 & 1737 & 1739 & 1765 & 1790 \\
\hline Terrebonne & & 238 & 258 & 318 & 394 & 360 & 976 & 1838 \\
\hline Ile-Jésus & & 655 & 687 & 653 & 708 & 742 & 2379 & 4653 \\
\hline Lachenaie & & 369 & 388 & 455 & 502 & 506 & 894 & 1783 \\
\hline Ensemble & 961 & & & & & & & \\
\hline Saint-Sulpice & & 643 & 669 & 755 & 578 & 858 & 1567 & 4268 \\
\hline Lavaltrie & & 153 & 167 & 212 & 171 & 248 & 327 & 1251 \\
\hline Ensemble & 545 & & & & & & & \\
\hline $\begin{array}{l}\text { Repentigny } \\
\text { L'Assomption } \\
\text { Blainville }\end{array}$ & 281 & 375 & 399 & 449 & 485 & 509 & 712 & $\begin{array}{r}1282 \\
1313 \\
974\end{array}$ \\
\hline Région & 1787 & 2433 & 2568 & 2842 & 2838 & 3223 & 6855 & 17362 \\
\hline Colonie & 25412 & 34042 & 35054 & 39177 & 39754 & 42925 & 69810 & 161311 \\
\hline
\end{tabular}

\section{TABLEAU 2}

TAUX DE CROISSANCE ANNUELle DE LA POPULATION TERREBONNE ET LA RÉGION, 1721-1790

(EN \%)

Années Terrebonne Ile-Jésus Lachenaie Repentigny St-Sulpice Lavaltrie Région Colonie

$\begin{array}{lllllllll}1730-39 & 4,7 & 1,4 & 3,5 & 3,5 & 3,2 & 5,5 & 3,1 & 2,6 \\ 1739-65 & 4,0 & 4,6 & 2,2 & 1,3 & 2,3 & 1,0 & 2,9 & 1,9 \\ 1765-90 & 4,3 & 4,6 & 4,7 & 4,0 & 6,9 & 9,3 & 6,4 & 5,7 \\ 1730-90 & 3,5 & 3,3 & 2,7 & 2,1 & 3,2 & 3,5 & 3,3 & 2,7\end{array}$

Source: tableau 1. 
aux années 1739-1765, ou, comme l'île-Jésus, terroir pratiquement plein au début des années $1780^{17}$, maintiennent leur rythme, Terrebonne, quoique en progression et dépassant maintenant en chiffres absolus les populations des autres seigneuries laïques, reste à la traîne du mouvement avec une légère avancée de $0,3 \%$.

Cette situation est le corollaire de la lente progression de l'occupation du sol dans le temps. D'ailleurs, à Terrebonne, si l'on en croit le mouvement des concessions et l'évolution du nombre de tenures ${ }^{18}$, la population de la zone agricole aurait augmenté presque exclusivement par accroissement naturel à partir des années 1770 .

Toutefois, un village y a vu le jour dès les années 1720 , ce qui est exceptionnel et donne à la seigneurie son trait distinctif: il n'existe en effet vers 1760 que six villages dans la colonie ${ }^{19}$. Au cours des années 1750 , le bourg est en pleine effervescence (au contraire de la zone agricole) et continuera dorénavant sur sa lancée comme s'il avait acquis son impulsion une fois pour toutes. Après 1765, en effet, l'agglomération s'étend aux dépens de la zone agraire et moins à l'initiative de la seigneuresse, Élisabeth de Ramezay, veuve La Corne, qu'à celle de possesseurs de terres avantageusement situées à proximité ou à l'intérieur des limites du village. C'est ainsi que, entre 1765 et 1775 , Jean-Baptiste Dupré, avec l'autorisation de madame de Ramezay, lotit la terre qu'il possède, adossée au village. Il y découpe 23 emplacements qui se vendent rapidement: en 1772, 17 sont déjà passés entre d'autres mains. De plus, les curés Jean-Baptiste Maisonbasse et Antoine Proulx morcèlent un vaste terrain que se transmettent depuis 1730 les détenteurs de la cure de Terrebonne. Quatre parcelles sont

17. Sylvie Dépatie et al., Contributions..., 21.

18. Qui a marqué un ralentissement dans les années 1740 et 1750 et n'a plus bougé entre 1772 et 1781 . Voir le chapitre sur la colonisation dans notre mémoire de maitrise pour des données chiffrées et une analyse de la question.

19. Il s'agit de Charlesbourg, Laprairie, Boucherville, Terrebonne, Pointe-aux-Trembles et Verchères. Mais, d'après la carte de Murray, il y a au Canada, autour de 1763, 14 agglomérations de 10 maisons et plus dont 8 dans le gouvernement de Montréal. Richard Cole Harris, The Seigneurial System in Early Canada. A Geographical Study (Kingston, McGill-Queen's University Press, 1984) (1966), 176; Serge Courville, Entre ville et campagne. L'essor du village dans les seigneuries du Bas-Canada (Sainte-Foy, Les Presses de l'Université Laval, 1990), 24. À l'île Jésus, le premier bourg n'apparaitra qu'au dernier quart du XVIII ${ }^{e}$ siècle et celui de l'Assomption, dans la seigneurie de Saint-Sulpice, est encore, en 1765, à l'état «embryonnaire». Paul Labonne, Structuration de l'espace et économie villageoise: deux études de cas: SaintMartin de l'île Jésus et l'Abord-à-Plouffe (1774-1861), mémoire de M.A. (Histoire), Université de Montréal, 1994, 31; Lise St-Georges, "Commerce, crédit et transactions foncières: pratiques de la communauté marchande du bourg de l'Assomption, 1748-1791", Revue d'histoire de l'Amérique française, 39,3 (hiver 1986): 325. 
ainsi vendues entre 1756 et $1766^{20}$. Au tournant des années 1770 , le nombre de lotissements occupés au village approche la soixantaine et une cinquantaine de maisons y sont construites et habitées ${ }^{21}$. Dorénavant, le bourg absorbe la plus grande part de l'expansion démographique de la seigneurie et, pendant les quelques décennies à venir, la zone agricole continuera à ne se développer que lentement ${ }^{22}$.

La croissance précoce du village s'explique par la présence des moulins qui sont au cour de la vie économique de Terrebonne, la minoterie plus particulièrement, car elle génère plus d'activités. La circulation des grains et des gens engendre naturellement la multiplication des services à l'origine de son essor. Ce sont, en effet, les surplus de la région dans son ensemble et pas seulement, ni même principalement, la production des familles de Terrebonne, qui alimentent, par l'intermédiaire des marchands, la minoterie.

\section{L'ENTREPRISE}

Elle consiste en un moulin marchand à deux, trois ou quatre moulanges et un ou deux moulins à scie, selon le moment. En 1738 viennent s'y ajouter les bâtiments d'une forge que l'abbé Lepage et les cousins d'Ailleboust sont en train d'implanter à Terrebonne. Ce projet, quoique fort avancé alors, ne verra finalement pas le jour. Mais les pertes encourues par ses promoteurs auront des conséquences néfastes sur les autres secteurs de l'entreprise et c'est pourquoi nous devons en tenir compte.

Lorsqu'on aborde l'étude de l'entreprise seigneuriale à Terrebonne, on en vient tout naturellement à tracer une ligne de démarcation entre les périodes de l'administration Lepage (1720-1744) et celle de la famille La Corne - père, mère et fils - qui, elle, s'étend de 1744 à 1775 . D'abord parce qu'au transfert de propriété

20. Terrier de Terrebonne, Archives privées de monsieur Henri Masson. Ventes d'emplacement: à Pierre Limoges, notaire C.-F. Coron, 2 juin 1756; à Michel Minville, ibid., 3 janvier 1759; à Antoine Dumas, ibid., 24 septembre 1761; à François Coursel(?), notaire Antoine Foucher, 19 août 1766.

21. Solange De Blois, Possibilités et limites..., 58-60.

22. En 1781, le nombre de maisons dans la zone agricole est encore bien en deça du nombre de tenures, c'est-à-dire du nombre de parcelles effectivement découpées sur le sol par l'arpenteur: 170 contre 206 (le nombre de tenures n'a pas bougé depuis 1772). La population habitant la Mascouche de Terrebonne et les Plaines, qui formera en 1786 la nouvelle paroisse de Sainte-Anne des Plaines, augmente tout doucement entre 1765 et 1790, passant de 436 à 724 . Pendant ce temps, celle de la côte de Terrebonne, paroisse de Saint-Louis, territoire pourtant saturé mais où se trouve le village, passe de 540 à 1114 . Solange De Blois, Possibilités et limites..., 29; P.-G. Roy, «Le recensement du gouvernement de Montréal (1765)», Rapport de l'archiviste de la Province de Québec pour 1936-1937; Canada, Recensement du Canada - 18701871, IV: Recensements du Canada, 1665 à 1871 (Ottawa, 1876). 
correspond un changement de conjoncture: 1744 marque le début de la guerre de Succession d'Autriche avec sa conséquence inévitable, un complet bouleversement des conditions des échanges. Mais aussi parce que les seigneurs qui se succèdent à Terrebonne conduisent leurs affaires de manière très dissemblable et que celles-ci connaîtront des destins différents. L'ère Lepage s'achève sur une faillite totale; en contrepartie, sous la direction des La Corne, l'entreprise semble générer suffisamment de profits pour supporter les investissements et laisser des bénéfices nets.

Nous tracerons, pour chacune des sous-périodes, un portrait du seigneur et tâcherons de faire la part des choses entre sa manière d'opérer, sa stratégie face aux possibilités offertes par les marchés du bois et des grains, et la conjoncture, en tant que facteurs influençant sa réussite ou son échec.

\section{L'ère Lepage: 1720-1744}

\section{Le seigneur}

Louis Lepage achète la seigneurie de Terrebonne en 1720 à François-Marie Bouat (un marchand montréalais) qui l'avait acquise en 1718 et n'aura laissé dans l'histoire de Terrebonne d'autre trace que sa signature au bas des contrats de vente qui ont ratifié les deux transactions. À l'encontre des trois propriétaires précédents, Lepage va, à partir de 1721, résider dans son fief. Curé de la paroisse de SaintFrançois de Sales de l'île-Jésus et prêtre missionnaire de Terrebonne et Lachenaie, il a été fondé de pouvoir du séminaire de Québec pour l'île Jésus où il habitait jusque-là ${ }^{23}$. Il sera soupçonné par la suite d'avoir profité de la situation pour détourner des fonds et d'avoir comploté avec monsieur de Varennes (procureur du séminaire de 1707 à 1726) qui lui aurait prêté de fortes sommes d'argent ${ }^{24}$.

23. Pouvoir à Louis Lepage, contrat enregistré au greffe au printemps 1719 mais effectif depuis le 7 octobre 1718, notaire Michel Lepailleur, 27 avril 1719.

24. Il s'agit de Jean-Baptiste Gaultier de Varennes dont la gestion apparaissait «ruineuse» aux yeux des supérieurs du séminaire de Paris. L'abbé Jean-Henri Tremblay écrit de Paris en 1728 aux messieurs du séminaire de Québec, à propos des dettes qu'ils ont accumulées et des difficultés financières dans lesquelles ils se trouvent, qu'elles sont attribuables «à la mauvaise administration de ceux qui ont été chargés du temporel du séminaire». Il ajoute: "On ne saurait comprendre comment un $\mathrm{Mr}$ Lepage qui n'avait rien de sa famille aye pu acheter une terre comme celle qu'il a achetée et y aye fait comme on le dit pour 20,000 écus [soit 60000 livres tournois] de dépense, s'il n'avait détourné à cela les revenus de l'Isles-Jésus et n'avait pu s'entendre avec Mr de Varennes pour lui fournir des sommes qui ont causé l'augmentation de plus de 50000\# de dettes dont le séminaire se trouve accablé...» Honorius Provost, «JeanBaptiste Gaultier de Varennes», Dictionnaire biographique du Canada, 2: 249. «Mémoire de monsieur l'abbé Jean-Henri Tremblay au Messieurs du Séminaire, 1728», Archives du Séminaire de Québec, Lettre M, no 59. 
Fils de paysans, Louis Lepage est né à l'île d'Orléans en 1690 . Son père, René Lepage, acquiert en 1696 la seigneurie de Rimouski en échange d'une terre (située dans l'île) dont a hérité sa femme, Madeleine Gagnon ${ }^{25}$. C'est dire à quel point la seigneurie de Rimouski ne vaut pas cher. À partir de là, les Lepage essayent de créer l'illusion qu'ils ont acquis leurs lettres de noblesse.

René, en effet, du moment où il devient propriétaire du fief de Rimouski, s'affuble du titre de Sieur de Sainte-Claire, accolant à son patronyme le nom d'une de ses terres. Ses fils perpétueront la fable en adoptant les particules de Saint-Barnabé, pour Pierre, l'aîné et héritier de la moitié de la seigneurie; de Sainte-Claire pour Louis, destiné, comme un deuxième fils de famille, à la prêtrise (il sera ordonné en 1715); de Saint-François pour Germain, et ainsi de suite...

Le caractère entreprenant de Louis Lepage s'explique en grande partie par ses aspirations sociales. Il est conscient qu'en dehors de Rimouski (où à la mort de son père seules trois familles sont installées) il ne peut prétendre à un statut quelconque hormis celui que lui confère son état ecclésiastique, qui n'est pas sans valeur socialement, mais ne suffit pas pour s'élever. Étant donné ses origines, seul l'argent, avec ses attributs de pouvoir et d'influence, est à même de lui apporter la considération qu'il recherche. Au début, Louis aura l'appui financier de ses frères Germain et Paul, sans doute animés d'une ambition semblable. Tous trois conjuguent leurs efforts, le mirage de la réussite les aiguillonnant. Mais Paul, très rapidement, se retire. Germain reste associé pendant trois ou quatre ans à l'entreprise de scierie mais, dès 1734 ou 1735, Louis assume à nouveau seul les frais et les risques occasionnés par l'exploitation des moulins ${ }^{26}$.

Terrebonne restera aux mains de l'abbé Lepage pendant près d'un quart de siècle, jusqu'en 1744. C'est sous sa gouverne que s'est développée l'infrastructure «industrielle» et commerciale qui en sera la marque.

Très ambitieux, le seigneur de Terrebonne a pris d'énormes risques pour tâcher de rentabiliser son bien, misant sans aucun doute sur les possibilités offertes par le régime seigneurial (entendons les privilèges qu'il légitime) et sur son bénéfice ecclésiastique, mais surtout en

25. «Notes sur les seigneuries du district de Rimouski, suite», Bulletin des recherches historiques, 17: 259.

26. Sans doute se sont-ils retirés parce que l'entreprise se révélait ruineuse. En 1731, Louis doit 6000 livres à Germain. Après avoir rompu l'associaton, ce demier exploitera son propre moulin à scie, dans son arrière-fief, sur la rivière Mascouche. Obligation de Louis Lepage à Germain Lepage, notaire J.-C. Raimbault, $1^{\text {er }}$ septembre 1731; dépôt de transaction entre Germain Lepage et Louis de La Corne, notaire L.-C. Danré de Blanzy, 6 mai 1749. 
s'endettant royalement ${ }^{27}$. La faiblesse de son capital initial est d'ailleurs le principal facteur de son échec.

\section{Gestion des moulins et circuits commerciaux}

Un moulin marchand (ou une minoterie, une meunerie) est une industrie de transformation qui correspond à une étape dans le processus de commercialisation des surplus agricoles. Comme toute industrie, les minoteries doivent s'approvisionner en matière première et composer avec les aléas du marché. Mais, au départ, tout entrepreneur est confronté à la question de l'investissement.

La nécessité de disposer d'un important capital pour se lancer dans une entreprise de minoterie explique que huit des dix moulins marchands identifiés par Louise Dechêne appartiennent à des communautés religieuses ou à de riches marchands de Québec ${ }^{28}$. Qu'un Louis Lepage, en comparaison presque désargenté (sa dîme et les rentes de Terrebonne lui rapportent au maximum, et potentiellement seulement, 1500 livres au moment de l'achat de la seigneurie ${ }^{29}$ ), se soit lancé, à titre individuel, dans cette sorte d'affaire est particulier et plutôt étonnant. Il fallait qu'il croie fermement à une avancée rapide de la colonisation et à une expansion importante et à court terme du marché des grains et farines, comme d'ailleurs de celui du bois ${ }^{30}$. Dans ce dernier

27. Il se lance résolument en affaires pratiquement sans capital. Lorsqu'il vend sa seigneurie, en 1744, elle n'est toujours pas payée et ses dettes s'élèvent à 55268 livres. Il a emprunté de tous côtés: à des marchands qui, est-ce un hasard?, font, pour la plupart, le commerce des blés et farines et, parfois aussi, celui du bois (Jean-Baptiste Hervieux, Pierre de Lestage, Ignace Gamelin, Antoine Poudret, Charles Nolan dit Lamarque, Jacques Gadois dit Maugé, François Poulain de Francheville); à des officiers civils (Jean Crespin, décédé, mais à l'époque conseiller au Conseil supérieur de Québec, monsieur de Boisclerc, grand voyer, monsieur DeLanouillier, sans doute Nicolas, contrôleur de la marine dans les années 1720, JeanVictor Varin de LaMarre, successeur du précédent); à la compagnie des Indes; au curé Jean Bouffandeau, etc. Voir l'«État de ce que moy Louis Lepage de Sainte-Claire prestre doit» joint à l'acte de vente de la seigneurie, notaire Louis-Claude Danré de Blanzy, 15 janvier 1745; compte entre Louis Lepage et François Poulain de Francheville, notaire M. Lepailleur, 15 avril 1722; obligation de Germain Lepage et Louis Lepage à Jean-Victor Varin de LaMarre, notaire J.-C. Louet, 26 février 1734.

28. Louise Dechêne, Le partage..., 34-35. Voir supra note 24, le commentaire de l'abbé Tremblay qui estime à 60000 \# les investissements de l'abbé Lepage dans sa seigneurie dans les années 1720, soit avant l'agrandissement de ses moulins. Une somme de cette importance, même si on suppute que seulement la moitié a été affectée à la construction des moulins, n'est pas à la portée du premier venu.

29. Ses dîmes cumulées lui rapportent environ 1400 livres et les rentes de Terrebonne à peine une centaine au prorata moyen de $8 \#, 5 \mathrm{~s}$. Solange De Blois, Possibilités et limites..., 22 et 91 .

30. Ses créanciers, quant à eux, devaient estimer bonnes ses chances de succès, puisque leurs prêts (en argent, effets et fournitures) servirent à financer la construction et l'agrandissement subséquent des moulins à farine et à scie. D'un autre côté, la seigneurie, une fois dotée de ses moulins, leur offrait une solide garantie et, effectivement, ils ont été remboursés après la 
secteur, il comptait sur les commandes du roi - surestimant, suivant l'optimiste Hocquart, les possibilités de croissance de la demande ${ }^{31}$ et il a eu le tort, à partir de 1727 , d'hypothéquer toute sa production par des contrats à long terme avec l'intendant. On imagine qu'il espérait retirer de confortables revenus de ses entreprises et éponger son déficit dans des délais assez rapprochés.

La mise sur pied d'un moulin à scie coûte peu comparativement à une minoterie ${ }^{32}$, mais les frais d'opération et les risques sont très élevés. Ainsi, il est rare qu'un individu - à part quelques marchands solidement établis comme Pierre de Lestage, qui place dans ses moulins des capitaux tirés du commerce des fourrures et s'est engagé dans le commerce local des grains et du bois dès les années 1720 , sinon avant - soit suffisamment bien nanti pour investir à la fois dans un moulin à farine et dans un moulin à scie.

Lepage constitue donc un cas tout à fait à part dans l'ensemble des minotiers et entrepreneurs en bois de sciage qui se lancent en affaire au cours des années 1710 et 1720 . Pour tous, ces affaires sont hasardeuses, mais pour lui, dépourvu de toute assise financière en dehors de sa seigneurie, elles deviennent périlleuses. Qu'à cela ne tienne!

\footnotetext{
vente de Terrebonne. Voir compte entre Louis Lepage et François Poulain de Francheville, notaire Michel Lepailleur, 15 avril 1722; obligation de Louis Lepage à Ignace Gamelin et cie, ibid., 24 janvier 1727; obligation de Germain Lepage et Louis Lepage, associés, à Jean-Victor Varin de LaMarre, notaire J.-C. Louet, 26 février 1734; obligation de Louis Lepage à Charles Nolant dit Lamarque (pour un compte datant de 1733), notaire J.-B. Janvrin-Dufresne, 27 mars 1740. Tous ces actes révèlent que les sommes prêtées ont été affectées à la construction, à la réparation ou à l'agrandissement des moulins.

31. À l'origine de l'agrandissement de son moulin à scie au début des années 1730, se trouve le projet d'implantation d'un chantier naval royal à Québec, projet soumis au ministre par Hocquart en 1731. Suivant les plans de l'intendant, Lepage et Sabrevois de Bleury seraient les fournisseurs attitrés des chantiers. Ils pouvaient s'attendre à fournir, ensemble, pour au-delà de 50000 livres de bois d'œuvre pour la construction d'une seule flûte de 500 tonneaux. Maurepas refusera de souscrire au projet pour de bonnes raisons qu'il n'expose (évidemment) pas dans sa réponse à l'intendant: il a, cette année-là, renfloué l'entreprise des fournisseurs privilégiés des chantiers navals de Rochefort et Brest (les associés Babaud, Masson et Boesnier Duportal, entrepreneurs en bois de sciage, ses clients et protégés) en difficulté à la suite de la faillite de leurs banquiers parisiens. (En 1730 et 1731, les grandes places du négoce, en France et à l'étranger, sont en crise commerciale et financière.) Au total, le ministre de la Marine leur a avancé 157837 livres. Il récidivera l'année suivante, en 1732, accordant à la compagnie un prêt sans intérêt de 112000 livres. Le chantier naval royal de Québec ne verra finalement le jour qu'en 1739. Hocquart à Maurepas, «Projet de construction d'une flûte de 500 tonneaux», 30 septembre 1731, AC, C11A, volume 55, f. 15-25. Paul Walden Bamford, Privilege and Profit. A Business Family in Eighteenth-Century France (Philadelphia, University of Pennsylvania Press, 1988), 42. Charles Carrière, Négociants marseillais au XVIII siècle (Marseille, Institut historique de Provence, 1973), 1: 441.

32. Un moulin à scie de pièce sur pièce (soulignons que les moulins à scie sont toujours faits de bois), de dimensions courantes, soit environ 30 sur 25 pieds, coûte entre 1000 et 2000 livres. Voir les exemples cités dans Solange De Blois, Possibilités et limites..., 76-77.
} 
Lepage s'y jette malgré tout, faisant grande confiance au roi en tant que soutien et promoteur de l'industrie coloniale. En chemin, il s'est endetté de manière outrancière, comptant finir par tirer son épingle du jeu. Mais les dettes limitent la liberté de mouvement d'un entrepreneur; et leur trop grande accumulation, de toute évidence, a des conséquences sur ses méthodes de gestion et le succès de ses affaires. Lepage exploitera toujours directement ses moulins. Il ne pouvait certainement pas se permettre de laisser aller une part des revenus à un fermier ou à un associé ${ }^{33}$. D'un autre côté, l'état de ses finances et le fait qu'il ait aliéné la production de sa scierie par des marchés contraignants avec le roi ne faisaient pas de lui un partenaire très attrayant pour un associé potentiel ${ }^{34}$. Cette situation qui, à première vue, peut paraître avantageuse, rend au contraire l'entrepreneur excessivement vulnérable puisqu'il assume alors seul tous les risques. Or, du côté de l'offre plus particulièrement, les marchés des grains et du bois sont tout ce qu'il y a de plus aléatoire à l'époque car, tout comme l'agriculture, la production de moulins actionnés par la force hydraulique dépend fortement des conditions atmosphériques.

Il suffit d'un léger fléchissement des récoltes pour que l'ensemble des surplus paysans baisse considérablement, diminuant d'autant les

33. La plupart, pour ne pas dire tous les exploitants de moulins marchands, afferment leur entreprise à partir des années 1730, alors que la production agricole et le commerce extérieur prennent toujours plus d'expansion; et les scieries d'une certaine envergure sont gérées par un associé (généralement un marchand) qui partagent pour moitié les profits et pertes. C'est seulement à partir des années 1750 que l'affermage de moulins à scie devient pratique fréquente, signe que l'industrie du bois est devenue plus lucrative et ce, malgré l'embargo sur les exportations décrété par Bigot en 1756. La demande intérieure s'est en effet accrue considérablement. Ibid., 147-149.

34. Les livraisons de Lepage sont exclusivement destinées au chantier naval royal de Rochefort. Les marchés qu'il conclut l'obligent à livrer des quantités considérables de bois (eu égard aux difficiles conditions de production de l'époque) de dimensions et de types bien définis, et s'avèrent souvent trop exigeants pour les possibilités d'un entrepreneur. Par le contrat qu'il signe en 1727, par exemple, Lepage s'engage à fournir, par année, pendant cinq années consécutives, 600 bordages de fresne, chêne et pin, et 5000 planches de pin de différentes longueurs. De plus, le transport du bois à Québec est aux frais et aux risques de l'entrepreneur. Comme il y a loin de l'arbre à la scie (séparés par toute une série d'opérations) et de Terrebonne à Québec, que les contraintes liées au climat et la difficulté de trouver des chênes et des pins de dimension suffisante à distance raisonnable compromettent considérablement le succès des fournisseurs du roi de la région de Montréal, il est presque inévitable qu'un entrepreneur prenne, à un moment ou à un autre, du retard dans ses livraisons. Et ce sera le cas de Lepage, comme nous le verrons. D'ailleurs madame de Ramezay, veuve du gouverneur de Montréal et fournisseur de l'État avant Lepage, a renoncé à renouveler son contrat en raison des pertes, importantes et répétitives, encourues par son entreprise de sciage. Marché entre Louis Lepage et Claude-Thomas Dupuy, intendant, notaire J.-C. Louet, 12 octobre 1727. Joseph-Noël Fauteux, Essai sur l' industrie..., 1: 209. Les associés potentiels des entrepreneurs sont des marchands. Or ce qui les intéresse d'abord, c'est le commerce local (le transport du bois à distance est par trop risqué et onéreux), qui est restreint, mais qui existe. Produire pour le roi, dans les conditions que nous venons de décrire, interdit, à toutes fins utiles, l'accès à ce marché. 
revenus des moulins marchands ${ }^{35}$. Car ce sont les grains de surplus dont disposent les paysans - après avoir nourri leur famille, payé leur dîme et leurs redevances, prélevé la part des semences de l'année à venir - qui entrent dans le circuit des échanges et qui, de plus en plus abondants à partir des années 1730 , bien que toujours fluctuants, alimentent le commerce des grains et farines et fournissent leur matière première aux meuneries.

Différents réseaux d'échange coexistent à l'intérieur de l'industrie céréalière: entre producteurs et marchands, marchands et boulangers, fermiers de moulin ou minotiers et boulangers ou marchands, ou encore entre marchands et négociants, pour ne citer que les plus importants. Le moulin à farine de Terrebonne est à la croisée de tous ces circuits $^{36}$. Les grains qu'il transforme ${ }^{37}$ proviennent de trois sources. Il s'agit des stocks amassés par le seigneur de Terrebonne en tant que propriétaire de fief et en tant que minotier; des blés apportés, très occasionnellement à l'époque de l'abbé Lepage, par les commis des magasins du roi; et, enfin, ceux des marchands qui, à eux seuls, constituent au moins $80 \%$ de l'apport total. Ce sont ces derniers qui nous intéressent plus particulièrement.

Le moulin de Terrebonne attire la clientèle de marchands équipeurs et exportateurs de Montréal et de Québec qui collectent, directement ou indirectement, des grains dans toute la région pour les expédier, une fois transformés, à Louisbourg et aux Antilles ${ }^{38}$. Citons pour exemple, Joseph Guyon Després qui achète en 1725 du blé pour la somme de 300 livres à Pierre Limoge, habitant aisé de Terrebonne $^{39}$. Gamelin et Lamarque, de Montréal, et Nicolas Philibert, de Québec, avaient des agents à Lachenaie ${ }^{40}$.

35. Louise Dechêne, Le partage..., 22.

36. Déjà en 1729 , ce sont plus de $\mathbf{2 0} 000$ minots de blé qui passent sous ses meules au cours de l'hiver et du printemps. Louise Dechêne, Le partage..., 34.

37. Ne nous concernent pas ici les grains consommés par la population de la seigneurie et qui sont assujettis à la banalité, mais seulement ceux qui sont commercialisés à l'échelle régionale. De toute façon, les revenus de la banalité sont peu importants (185 minots en 1739) en comparaison de ceux retirés de la transformation du blé amené au moulin par les marchands (entre 1200 et 1500 minots par année, en moyenne, à la même époque). Solange De Blois, Possibilités et limites..., 95 et 98.

38. Aux îles n'est exportée que de la fine fleur de farine, c'est-à-dire de la farine de la plus haute qualité, ayant nécessité un passage au crible. Une dizaine de moulins marchands en sont dotés au début des années 1730 dans les régions de Québec et de Montréal, dont celui de Terrebonne. Il n'est pas certain que les cribles des autres moulins marchands aient été actionnés mécaniquement.

39. Obligation de Joseph Guyon Després en faveur de Pierre Limoge, notaire J.-C. Raimbault, 21 octobre 1727. La transaction a eu lieu en 1725.

40. Louise Dechêne, Le partage..., 244, note 71. 
Toutefois, les marchands des villes accumulaient leurs stocks de céréales principalement par l'intermédiaire des marchands ruraux amasseurs de grains qui leur livraient de substantielles quantités de blé en retour des marchandises qu'ils leur livraient régulièrement pour alimenter leur commerce de détail. Ainsi, Pierre Ranger, de Rivièredes-Prairies, s'engage à payer à Jean Soumande, sur deux ans, la valeur de 4500 livres en blé et en pois, somme qui correspond à $75 \%$ de sa facture, et équivaut, «au cours du commerce», à une quantité de 1000 minots de blé. Joseph Chaumont, quant à lui, s'oblige à livrer près de 900 minots de blé à son fournisseur de Québec ${ }^{41}$. Ces marchands exportateurs de Montréal ou de Québec utilisaient vraisemblablement le moulin de Terrebonne, de loin le meilleur de la région.

Mais les principaux utilisateurs du moulin de l'abbé Lepage sont les marchands ruraux «spécialisés» dans le commerce des blés et farines qu'ils exportent en grandes quantités, sous forme de biscuit et de farines entières, à l'île Royale. Ils vendent aussi à d'autres marchands et à des boulangers. Eux non plus ne se contentent pas des retours que leur commerce de détail leur rapporte. Ils achètent directement des producteurs qui ont des surplus à écouler. C'est aussi à eux que les divers agents de commercialisation, seigneurs, curés, fermiers vendent les stocks qu'ils accumulent chacun de leur côté.

Pierre Ranger, dont nous venons de faire la connaissance, est le plus gros de ces marchands de grains locaux. Propriétaire d'une boulangerie, il fréquente le moulin de l'abbé Lepage et celui de l'île Jésus. Il transige avec la population des seigneuries de la rive nord, dont Terrebonne, et fournit Lepage en marchandises, contre farine ${ }^{42}$.

D'autres marchands des paroisses avoisinantes, François Poisset lui aussi de Rivière-des-Prairies, Charles-Auguste Réaume, qui s'installe à l'île Jésus en 1734, et Pierre Papin, arrivé à l'île Jésus en 1738, sont tous actifs dans le commerce des produits agricoles et faisaient sans doute transformer une partie des blés qu'ils collectaient au moulin de Terrebonne, ne serait-ce que pendant les périodes où celui de l'île était défectueux ou arrêté par les glaces, ou par une pénurie d'eau, ce qui était relativement fréquent ${ }^{43}$. Le fait que Papin quitte l'île Jésus

41. Convention entre Pierre Ranger et Jean Soumande, notaire J.-B. Adhémar, 22 septembre 1729; obligation par Joseph Chaumont, marchand de Terrebonne, à Claude Leblond, négociant de Québec, notaire François Simonnet, 8 octobre 1763. Ce procédé est courant tout au long du siècle et au-delà.

42. ANQM, Registre des audiences, 06-M, T601-2/16, f. 307, 31 janvier 1738, Ranger contre Catherine Belisle, veuve de Barthélémy Sicard, boulanger; ibid., f. 110, 16 août 1737, Pierre Ranger contre Pierre Maisonneuve, habitant de Terrebonne; transport de rente à Ranger par Louis Lepage, notaire C.-F. Coron, 10 janvier 1737.

43. Sylvie Dépatie, L'évolution..., 115; Sylvie Dépatie et al., Contributions..., 44. 
en 1741 pour s'établir à Terrebonne où il achète la maison et l'emplacement de François Simon Delorme, ancien meunier de l'endroit, y construit des hangars et se procure un bluteau, renforce cette hypothèse qui par ailleurs va dans le sens de la logique des choses ${ }^{44}$. Après tout, le moulin de Terrebonne est facilement accessible à ces gens qui possèdent des bateaux (sauf Papin) et il a le mérite d'être fiable.

Alexandre d'Ailleboust de Cuisy, qui s'est fixé à Terrebonne en 1739 pour exploiter le moulin à scie de l'abbé Lepage (nous y reviendrons), a lui aussi laissé des traces de son activité de marchand de grains. En attendant de construire son propre hangar et une maison de pierre avec grenier et magasin, il entrepose dans les greniers du moulin de Terrebonne des sons (c'est-à-dire des grosses farines) fabriqués à partir des grains qu'il recueille localement. Notons qu'il fait aussi du commerce de détail. Il possède un bluteau et vend donc lui aussi des farines. De plus, il a loué à Jean-Baptiste Côté un hangar à Lachenaie, «assis sur la terre de Charles Mathieu», marchand du lieu, où il entreposait les grains qu'il collectait à l'est de Terrebonne ${ }^{45}$.

Tous ces marchands exportent farines et biscuits, mais ils écoulent aussi une partie de leurs produits dans la colonie. En plus de fournir le marché extérieur, les farines qui sortent de Terrebonne pourvoient en effet à la demande des villes ${ }^{46}$. Dans une moindre mesure en temps de paix, elles répondent aussi à la demande des magasins du roi (dont François Poisset est un bon fournisseur et Lepage un fournisseur occasionnel $^{47}$ ) qui, possédant leurs propres boulangeries jusqu'en 1745, apportent au moulin les grains qu'ils ont achetés à des marchands et minotiers.

L'industrie forestière coloniale est plus ancienne que la meunerie d'un bon demi-siècle ${ }^{48}$, mais le marché du bois de sciage est plus étroit que celui des céréales. Cette situation est liée autant à des éléments de structure, aux exigences du commerce lui-même, qu'à des facteurs

44. Vente d'un emplacement par François Simon Delorme à Pierre Papin, notaire C.-F. Coron, 26 juillet 1741; vente d'emplacement par Pierre Papin à Joseph Durocher, ibid., 13 mai 1747.

45. ANQM, Registre des audiences, 06-M, T601-2/18, f. 170, 4 décembre 1739, de Cuisy contre Lepage; vente d'un emplacement par Alexandre d'Ailleboust de Cuisy à Louis de La Corne, notaire L.-C. Danré de Blanzy, 8 mars 1751.

46. Les principaux acheteurs de farine des villes sont les boulangers qui fabriquent le pain d'une partie des citadins et, sur commande, le biscuit pour l'armement des navires et l'exportation, les voyages de traite et les pêcheries. Louise Dechêne, Le partage..., 89-96.

47. Solange De Blois, Possibilités et limites..., 124-125.

48. Des moulins à scie existent en Nouvelle-France dès le milieu du XVII ${ }^{\mathrm{e}}$ siècle; le plus ancien aurait appartenu aux jésuites. Du merrain est exporté en France dès 1653. Joseph-Noël Fauteux, Essai sur l'industrie..., 1: 172-173. 
conjoncturels. De plus, la demande est moins importante au cours des années Lepage qu'elle ne le sera par la suite.

Sur le plan intérieur, la population paysanne se suffit grosso modo à elle-même et seuls les besoins des habitants des villes, de l'industrie navale (jusqu'en 1739, la construction des bateaux est entièrement aux mains des particuliers), des travaux publics et des installations civiles et militaires sont à combler. Les exportations vers les Antilles sont contrôlées par des marchands de Québec et de Louisbourg. Les exportations vers la France sont le fait de contrats conclus directement entre l'intendant et les fournisseurs ${ }^{49}$ et ne représentent pas un débouché bien important. En fait, la métropole n'a pas besoin des bois canadiens ${ }^{50}$ et ce secteur ne prendra jamais d'expansion.

En dehors des marchés avec l'intendant, qui pourvoient aussi aux besoins de l'administration coloniale, les circuits d'échange locaux et régionaux sont les seules voies d'écoulement possibles pour la production des scieries du gouvernement de Montréal. Or, sous la gouverne de l'abbé Lepage, le moulin à scie de Terrebonne n'a qu'un seul client: le roi. C'est là, après l'endettement massif et toujours croissant du seigneur de Terrebonne, la seconde faiblesse de son entreprise.

Vers la faillite: le rôle décisif du projet des forges

Pour la première fois en 1732 , selon les sources, l'abbé Lepage ne pourra honorer son contrat de fourniture de bois à l'intendant, les glaces de l'hiver ayant empêché le moulin de fonctionner et les basses eaux de l'été d'expédier bordages et madriers à Québec ${ }^{51}$. Pour ajouter à ses malheurs, ses créanciers deviennent, à partir de 1733, de plus en plus pressants. On va jusqu'à la saisie des grains et des bois des moulins de Terrebonne à plusieurs reprises (et de ses autres biens

49. Des navires marchands français chargent des bois pour la France, mais ces sorties restent marginales dans l'ensemble des exportations de produits forestiers. Voir le tableau des exportations forestières de Jean A. Lunn, dans Développement économique de la NouvelleFrance (Montréal, 1986), 315-317.

50. La France peut très bien se passer de cette source d'approvisionnement pour ses arsenaux. Durant les années 1730 et 1740 , les chantiers navals royaux français sont approvisionnés en bordages et bois longs de toutes sortes au-delà de leurs besoins, Babaud et Cie, fournisseurs exclusifs de la marine, y livrant régulièrement plus que ce qui était stipulé dans leurs contrats. Le pays n'est pas encore à court de ressources forestières ni d'ailleurs la Baltique, d'où il importe les mâts dont il a besoin, moins coûteux que ceux du Canada si on considère le prix du transport à travers l'Atlantique, et de meilleure qualité, semble-t-il. Jean A. Lunn, Développement..., 147. Paul Walden Bamford, Privilege and Profit..., 43, 58-59, 71. Voir aussi les pages que consacre Bamford aux difficultés et aux coûts de transport de même qu'à la qualité des mâts importés du Canada par la mère patrie, dans Paul Walden Bamford, Forests and French Sea Power, 1660-1789 (Toronto, University of Toronto Press, 1956), 119-127.

51. Hocquart à Maurepas, 18 octobre 1732, AC, C11A, volume 57, f. 235-236. 
meubles apparemment) pour se faire payer. Et souvent les montants dus seront remboursés, en tout ou en partie, en planches ${ }^{52}$. Il n'est pas étonnant dans ces conditions que Lepage n'ait pu satisfaire au nouveau marché qu'il a conclu avec le roi en 1735 et ce, ni en 1736, ni en 1737 , ni en 1738 . Occupé à scier des planches pour payer ses dettes, le seigneur de Terrebonne accumule toujours plus de retards dans ses livraisons au roi. En somme, à partir de 1732, il n'a pour ainsi dire plus retiré de revenus de son moulin à scie ${ }^{53}$.

Par ailleurs, il est presque certain qu'en 1738, la scierie a été monopolisée pour les besoins de l'entreprise des forges. Cette annéelà, Louis Lepage a formé une société avec les cousins d'Ailleboust (Louis d'Ailleboust de Coulonge, Antoine d'Ailleboust de Mantet et Alexandre d'Ailleboust de Cuisy) pour l'établissement et l'exploitation d'une forge à Terrebonne ${ }^{54}$. Il a cédé à perpétuité à ses associés tous ses droits sur les mines pouvant se trouver dans le sous-sol de la seigneurie, en échange de quoi de Cuisy et de Mantet ont avancé les fonds exigés par la mise sur pied et l'exploitation de l'entreprise. Lepage devait fournir tout le bois nécessaire à la construction des bâtiments de la forge. Au bout du compte, les d'Ailleboust ont déboursé 50000 écus, soit 150000 livres, ce qui représente une somme considérable. Au moment où Hocquart interdit à Lepage de continuer ses ouvrages (en août 1738), le projet était si bien avancé qu'il ne «restait plus que les soufflets à poser ${ }^{55}$ ». C'est dire que le moulin à scie a largement été mobilisé au profit de la société des forges au cours des mois précédents.

Hocquart, qui à l'origine avait soutenu l'entreprise, a nié après coup avoir jamais encouragé les associés ${ }^{56}$. Il faut dire qu'il a désor-

52. C'est le cas pour les créances de Jean-Eustache Lanoullier de Boiscler, grand voyer et conseiller du roi, de Jean Bouffandeau, curé de Terrebonne en 1734-1735 et du séminaire de Québec, entre autres. Voir Solange De Blois, Possibilités et limites..., 154-156.

53. Même auparavant les choses n'étaient pas simples, le roi pouvant se montrer client fort capricieux. La quantité à livrer était parfois modifiée à la baisse par l'intendant qui dérogeait ainsi au contrat établi avec l'entrepreneur. C'est ce qui arrive à Lepage en 1729. Et même une fois les planches, madriers et bordages livrés à Québec, il pouvait arriver que l'intendant les refuse, parce que de mauvaise qualité ou mal débités. Hocquart au ministre, 25 octobre 1729, AC, C11A, volume 51, f. 248. Idem, 19 octobre 1733, AC, C11A, volume 60, f. 141.

54. Société entre Louis Lepage et les sus-nommés, notaire Louis-Claude Danré de Blanzy, 12 juillet 1738.

55. Mémoire de Louis Lepage de Sainte-Claire au ministre, joint à sa lettre du 20 octobre 1740, AC, C11A, volume 111, ff. 38-39.

56. Dans son mémoire de 1740 , Lepage déclare au ministre que, bien que l'intendant prétende ne pas l'avoir encouragé dans son projet, il lui avait bel et bien donné son appui. Hocquart a bien dû promettre quelque chose: il est difficile de comprendre, autrement, comment les cousins d'Ailleboust, qui ne sont pas nés de la dernière pluie, auraient investi autant d'argent dans l'entreprise. Dans la lettre qu'il adresse au ministre en 1738, Lepage fait référence à une 
mais des intérêts dans les forges de Saint-Maurice ${ }^{57}$ et qu'en 1737 et 1738, il était fort occupé à convaincre Maurepas de supporter ces dernières, à coups de subsides. Il y réussit d'ailleurs ${ }^{58}$. Il n'y a donc rien de surprenant à ce que le roi, déjà bailleur de fonds d'une compagnie en difficulté qui lui coûte de plus en plus cher, ait rejeté le projet concurrent des Lepage et d'Ailleboust ${ }^{59}$.

Le moins que l'on puisse dire est qu'ils se sont montrés bien téméraires en n'attendant pas la permission du ministre pour entreprendre leurs travaux ${ }^{60}$. Tout laisse croire pourtant qu'ils ont été, d'une manière ou d'une autre, abusés par le versatile intendant. Comme en 1731 - alors qu'il a agrandi son moulin à scie et commencé à préparer des bordages et des madriers pour alimenter un chantier qui n'existera encore longtemps que dans l'imagination de l'intendant Lepage a renoncé à toute prudence, anticipant sur les événements et s'engageant à fond dans un vaste projet aux bases peu solides. On peut se demander d'ailleurs à quel point cette audace de forcené n'est pas liée à son manque de liquidités et ne constitue pas un palliatif à la pauvreté de ses moyens financiers.

Mais cette fois le contrecoup est plus dur. Car, en compensation des avances faites par les d'Ailleboust et qui sont perdues pour eux, Lepage leur cède la jouissance de son moulin à scie pendant neuf ans, leur fournissant le bois par-dessus le marchébit. Tenace, le seigneur de

lettre de François-Étienne Cugnet dans laquelle ce dernier se dit prêt, aussi bien que FrançoisOlivier de Vézin (ils sont respectivement directeur et maître de forges à l'établissement de SaintMaurice) à investir dans l'affaire. «Mémoire pour les forges de Terrebonne», 20 octobre 1740, AC, C11A, volume 111, f. 38; Louis Lepage de Sainte-Claire au ministre, 18 octobre 1738, AC, C11A, volume 70, ff. 236-237.

57. Cameron Nish, François-Étienne Cugnet, 1719-1751: entrepreneur et entreprises en Nouvelle-France (Montréal, 1975), 63.

58. Le ministre s'est engagé en 1737 à faire une avance de 100000 livres à la nouvelle Compagnie des Forges de Saint-Maurice dont l'établissement avait commencé en mai 1736. En 1738, la somme due au roi a augmenté de 92000 livres. Le premier feu des forges fut allumé en août, au moment précis où Hocquart interdit aux associés de Terrebonne de poursuivre leur œuvre. Ibid., 69-71.

59. C'est d'autant moins surprenant que la Marine, grosse consommatrice de produits du fer pour l'armement, a en 1736 décidé, après avoir exploité à son compte les forges royales de Cosne, de les passer à l'entreprise privée, en l'occurrence celle de Babaud et cie qui a déjà le monopole des fournitures de bois aux arsenaux. Lepage et consorts tentent leur entrée dans ce secteur de l'industrie à un bien mauvais moment: Hocquart aussi bien que Maurepas avaient les yeux tournés ailleurs. Paul W. Bamford, Privilege and Profit..., 93.

60. Ici comme en France, l'exploitation des mines est prérogative royale. Aucun particulier ne peut, sans une permission spéciale du roi, extraire et tirer profit des métaux présents dans le sol, même des terres qui lui appartiennent.

61. Accord et convention entre Louis Lepage et Louis, Antoine et Alexandre d'Ailleboust, notaire François Simonnet, 29 septembre 1739. C'est de Cuisy qui prendra la direction de la scierie. 
Terrebonne ne renonce pas encore. Il arrivera à trouver l'argent nécessaire à la construction d'une deuxième scierie qu'il exploitera pour son compte sans réussir à se remettre à flot cependant. Car à toutes ses difficultés s'ajoutent les mauvaises récoltes successives de 1741, 1742 et 1743. Coup de grâce sans doute pour l'abbé qui se résout à vendre sa seigneurie aux dernières lueurs de l'été 1744 .

\section{L'ère La Corne: 1744-1775 (et au-delà)}

\section{Les seigneurs}

Louis de La Corne dit l'aîné, tirant avantage de la situation précaire de son prédécesseur, obtiendra la seigneurie de Terrebonne à très bon compte. Lepage a dû la laisser aller pour 60000 livres, montant qui dépasse à peine le total de ses dettes. Étant donné la hausse des prix de la propriété foncière de même que celle des revenus seigneuriaux de toute nature entre 1720 et 1744 , considérant de plus l'importance de ses investissements, cette somme parait bien faible.

Issu d'une famille de la petite noblesse militaire - son père, originaire d'Auvergne, est nommé sous-lieutenant en Canada en 1685 - La Corne est né à Montréal en 1696. Il a servi douze ans en France avant de revenir au Canada en 1732 pour s'y installer définitivement. Nommé lieutenant d'un détachement des troupes de la Marine en 1733 et capitaine en 1744, il devient chevalier de l'ordre de Saint-Louis en 1751. Il se retire de l'armée en $1758^{62}$. Il a été commandant de poste, à Michillimakinac et Carillon entre autres. Lui et ses trois frères, militaires eux aussi, se sont enrichis dans le commerce des fourrures. Marchands voyageurs ou commandants de poste en alternance, il arrivait qu'ils s'associent lorsque l'un d'eux était à la tête d'une garnison dans les pays d'en haut ${ }^{63}$. Leur patrimoine familial était presque inexistant, leur père n'ayant eu pour tout bien que sa solde d'officier et une petite portion de terre détachée du fief de Contrecour appartenant à la famille de sa femme.

Notre La Corne a épousé en 1740 Élisabeth de Ramezay, fille de Claude, de son vivant gouverneur de Montréal. Le couple aura cinq enfants dont quatre atteindront l'âge adulte. Les Ramezay sont une des 107.

62. Aegidius Fauteux, Les chevaliers de Saint-Louis en Canada (Montréal, 1940), 106-

63. Voir l'annexe I intitulée «Groupe central des marchands voyageurs, 1715-1750», dans François Gagnon, Marchands voyageurs et équipeurs de Montréal, 1715-1750, mémoire de M.A. (Histoire), Université de Montréal, 1995, xv; Madeleine Dionne-Tousignant et Pierre Tousignant, «Luc de La Corne dit La Corne Saint-Luc», Dictionnaire biographique du Canada, 4: 460 . 
familles les plus influentes de la colonie. Le père possédait déjà une belle fortune foncière que se sont partagée ses quatre enfants, en plus de se voir concéder, en 1739, des continuations qui doublent les superficies de leurs fiefs de Sorel et de Monnoir. Élisabeth de Ramezay est copropriétaire du quart des seigneuries de Ramezay et de Monnoir et du tiers de celle de Sorel ${ }^{64}$. Les Ramezay exploitent des scieries depuis deux générations déjà et c'est maintenant Louise, sœur d'Élisabeth, qui dirige l'industrie familiale.

La Corne, de son côté, ne possède pas de terre. Sa richesse est mobilière et provient des bénéfices de la traite. Pour lui, Terrebonne est un investissement et une source de profits supplémentaires. Nous avons là un deuxième exemple (après celui des d'Ailleboust) de transfert de capitaux du commerce des fourrures vers l'industrie. La Corne apporte de plus en mariage de solides, quoique indirectes, alliances avec deux des familles marchandes les plus en vue de Montréal. Deux de ses frères, Luc de La Corne et François-Josué de La Corne Dubreuil, ont épousé des filles de Jean-Baptiste Hervieux qui a fait fortune dans l'équipement pour la traite et le commerce de détail et d'exportations de farines et biscuit ${ }^{65}$. Le troisième (il s'agit de Louis dit le chevalier de La Corne) a convolé, en 1728, avec Marie-Anne Hubert de Lacroix, veuve de Jacques-Charles de Couagne. Cette dernière est encore alliée à cette grande famille de marchands par la sœur de sa mère, Marie Godé, deuxième femme de Charles de Couagne, père (toujours vivant) de son époux décédé. Ces liens profitent bien sûr aux autres Lacorne ${ }^{66}$ tout comme ceux établis par ces derniers avec les Hervieux et les Ramezay servent, à point nommé, les intérêts de tous les membres du clan.

En 1762, à la mort de Louis de La Corne, madame de Ramezay se charge de l'administration de la seigneurie jusqu'à ce que son fils aîné prenne possession de son héritage en 1772 . Louis, le nouveau seigneur, mourra à peine trois ans plus tard au fort Saint-Jean, des suites d'une maladie, et les survivants de la jeune génération, ses sœurs Marie-Louise-Charlotte et Marie-Angélique, et son frère Fran-

64. Pierre-Georges Roy, Inventaire des concessions en fief et seigneurie, foi et hommage et aveux et dénombrements, conservés aux archives de la Province de Québec (Beauceville, 1930-1932), 2: 169; 4: 197 et 218.

65. Louise Dechêne, Le partage..., 85; C. J. Russ, «Luc de Lacorne» et «François-Josué de Lacorne-Dubreuil», Dictionnaire biographique du Canada, 3: 357 et 359.

66. En 1739, un des frères La Corne, La Corne Dubreuil, est associé à un François de Couagne qui «prend soin de ses entreprises financières» à Montréal pendant qu'il se trouve à Michillimakinac comme commandant de poste en second. Peut-être ont-ils des intérêts communs dans le commerce des fourrures. C. J. Russ, «François-Josué de La Corne Dubreuil», Dictionnaire biographique du Canada, 3: 359. 
çois-Josué, se partageront la seigneurie. Terrebonne restera aux mains de la famille La Corne jusqu'en 1784 et aura pris une valeur considérable depuis son acquisition par l'abbé Lepage: Jacob Jordan, gros marchand de grains anglophone, la paiera 184000 livres.

Malgré tout, les Lacorne auront au total peu investi dans l'affaire en comparaison de ce que Lepage y avait engagé, et pas seulement sur le plan financier. Alors que ce dernier administrait lui-même ses entreprises, les premiers exploiteront leurs moulins, le plus souvent, par faire-valoir indirect ou en société à moitié profits et pertes, privilégiant l'une ou l'autre forme selon les conditions du marché, tout en jouant de leurs alliances familiales, politiques et commerciales. Deux styles tout à fait différents de gestion, tributaires, pour une part cela va de soi, de la conjoncture et de l'intérêt du capital marchand envers les industries meunière et forestière, intérêt qui grandit au diapason des possibilités de profits qu'elles offrent.

Gestion des moulins et marchés

Louis de La Corne a lui aussi dû emprunter pour payer sa seigneurie. Son frère, le chevalier, lui a en effet avancé 40000 livres, soit les deux tiers de la somme demandée par Lepage ${ }^{67}$. À peine trois mois plus tard, le chevalier réclame son dû (on ne connaît pas les raisons de ce revirement) et procède par saisie de la seigneurie. L'affaire est envoyée devant le Conseil supérieur mais sera finalement réglée à l'amiable en $1749^{68}$. Entre-temps, Lacorne a pu profiter de son acquisition et paisiblement encaisser les revenus de ses terres et de ses moulins.

Malgré les apparences - Lacorne a emprunté l'essentiel du capital investi dans son entreprise et s'est vu saisir son bien - la situation économique du nouveau seigneur n'a pas grand-chose en commun avec celle de l'ancien propriétaire du fief, en partie parce qu'il a fait un bon mariage. Le couple, on l'a vu, en plus de pouvoir tirer parti d'un réseau complexe d'alliances à divers degrés, a d'autres sources de revenus que Terrebonne et ses industries. Leur entreprise s'appuie sur une fortune foncière non négligeable quoiqu'elle appartienne en propre à Élisabeth de Ramezay. Des membres des deux familles ont des intérêts et ce, depuis des décennies, dans presque tous les secteurs de l'économie: industrie du bois, traite des fourrures, commerce des grains et autres denrées.

67. Obligation de Louis de La Corne l'aîné à Louis de Chapt, dit le Chevalier de La Corne, notaire L.-C. Danré de Blanzy, 18 janvier 1745.

68. Henri Masson, La seigneurie de Terrebonne sous le Régime français (Montréal, l'auteur, 1982), 137 et $145-146$. 
Non seulement la condition sociale, le niveau de fortune et le degré d'insertion des nouveaux propriétaires de Terrebonne dans les réseaux économiques (et politiques) de la colonie tranchent-ils sur ceux de leur prédécesseur, mais la conjoncture change du tout au tout en 1744. Les dépenses militaires de toute nature montent en flèche avec le début de la guerre de Succession d'Autriche. Dans le secteur des provisions de bouche, l'approvisionnement en céréales et autres denrées devient une activité très profitable pour ceux-là (parmi lesquels de nombreux nouveaux venus alléchés par la perspective de gains rapides) qui deviennent fournisseurs du roi, qu'ils soient marchands, seigneurs ou fermiers de moulins marchands ${ }^{69}$, un état n'excluant pas l'autre. L'un des spéculateurs apparus à la faveur de la nouvelle conjoncture, Joseph Durocher, réside à Terrebonne. Il disparaîtra en 1750, laissant 70000 livres de dettes. Il achète d'énormes quantités de blé qu'il fait moudre au moulin de La Corne avant de les bluter, mettre en quarts et livrer dans les greniers du roi ${ }^{70}$. La présence de tels individus dans la région (qui produit d'importants surplus comparativement à la moyenne coloniale ${ }^{71}$ ) a des conséquences bénéfiques sur le volume d'activité au moulin des La Corne.

La Corne lui-même passe de gros marchés de vivres avec l'État. Ainsi, entre octobre 1746 et août 1747, ce sont des années de récolte abondante, il a expédié 2996 minots de blé aux magasins du roi. Le tout, vendu à un prix variant entre 3 et 4 livres le minot, rapporte 11537 livres à Lacorne, sans compter les pois, la viande de boucherie et les autres denrées qui font monter la facture à 20649 livres $^{72}$.

À cette époque, les La Corne exploitent leur moulin à farine par faire-valoir direct, ce qui explique qu'ils disposent d'aussi grandes quantités de blé. Nous ne savons rien des modalités d'exploitation de la minoterie entre 1748 et 1756 . Les états de dépenses de la colonie sous l'administration Bigot (il est arrivé à Québec en 1748) nous faisant défaut, on ne sait pas non plus à quel rythme et en quelles quantités Louis de La Corne a effectué ses livraisons au roi par la suite. Cependant, au début de la guerre de Sept Ans, le moulin est affermé à Joseph Cadet, munitionnaire du roi, à des conditions extrê-

69. Voir Louise Dechêne, Le partage..., 83-89 et 142-152, pour un exposé sur l'activité des marchands de grains avant 1744 et les modifications que connaissent le commerce et la circulation des céréales entre 1744 et 1760 , sous la pression d'une consommation militaire croissante et incoercible et les effets des politiques de l'intendance.

70. Ibid., 151 et Solange De Blois, Possibilités et limites..., 110.

71. À titre indicatif, pour l'année 1739 , les surplus de la région (c'est-à-dire des seigneuries de Terrebonne, l'Île-Jésus, Lachenaie, Repentigny, Saint-Sulpice et Lavaltrie) représentent $60 \%$ de sa production totale alors que, dans toute la colonie, les surplus ne correspondent qu'à $47,2 \%$ de la production d'ensemble. Ibid., 97.

72. «Préparatifs de guerre», AC, C11A, volume 88, ff. 248-250 et 252-253. 
mement avantageuses pour son propriétaire. Le prix du bail, en effet, est fixé à 9000 livres par an, payables en partie d'avance ${ }^{73}$. Le moulin, qui ne tournera pour ainsi dire plus que pour les besoins de l'intendance, est géré par un commis de Cadet, le marchand Jacques Perras. À la mort de sa femme en 1760, Perras déclare un actif en argent et effets de 156000 livres. Ses dettes passives s'élèvent à 14000 livres et il n'avoue aucune dette active. Le moins qu'on puisse dire, c'est que l'affaire (et la guerre) lui ont rapporté. En 1762, il assume toujours la gestion du moulin à farine (et celle du moulin à scie) de madame de Ramezay ${ }^{74}$.

En 1765 Alexandre Dumas, un marchand exportateur de Québec, loue les moulins à farine et à scie au prix de 7000 livres par $a^{75}$. Pour ce marchand qui, en 1779, sera accusé d'accaparer le marché du blé ${ }^{76}$, il est avantageux de louer un moulin qui lui permette de profiter du bassin d'approvisionnement que constitue la grande région montréalaise et de moudre à peu de frais ses propres stocks de grains. D'autant plus que, depuis 1763 , les conditions du marché se sont améliorées. Un nouveau couloir d'exportation vers l'Europe méridionale s'est ouvert pour les céréales nord-américaines (les marchands de Philadelphie et de New York l'exploitent eux aussi) et ce commerce prendra de plus en plus d'importance à partir de $1770^{77}$. Cette annéelà d'ailleurs, Zachary McCaulay, un autre négociant de Québec, place un agent à Terrebonne.

Le moulin à farine de Terrebonne continuera à exercer un grand pouvoir d'attraction sur les marchands quelles que soient les possibilités offertes par le marché des grains. En 1788, en plein milieu d'une période prolongée d'instabilité des échanges, la minoterie de Jordan, propriétaire de la seigneurie depuis 1784 , est au deuxième rang au

73. Bail à ferme du moulin à farine de Terrebonne, notaire Pierre Panet, 31 décembre 1756.

74. Engagement de Jacques Perras par Joseph Cadet, notaire Pierre Panet, 27 mars 1757; inventaire des biens de la communauté entre feue Thérèse Guy et Jacques Perras, notaire Antoine Foucher, 17 juillet 1760; inventaire des biens de la communauté La Corne-Ramezay, notaire Charles-François Coron, 2 juin 1762.

75. Bail à loyer et argent des moulins à farine et à scie de Terrebonne à Alexandre Dumas, notaire Pierre Mézière, 3 mars 1765. 304.

76. David Roberts, «Alexandre Dumas», Dictionnaire biographique du Canada, V: 303-

77. Thomas Wien, Peasant Accumulation..., 298; Fernand Ouellet, «The Colonial Economy and the World Market, 1760-1850: the Trade of the St.Lawrence Valley", dans Jacques A. Barbier, dir., Economy, Class \& Nation in Quebec (Toronto, 1991), 215, 224-225. 
Canada pour sa production ${ }^{78}$. Les conditions du bail de 1775 témoignent elles aussi d'une grande activité à la minoterie: le moulin est affermé contre un loyer de 1000 minots et le tiers des moutures ${ }^{79}$. Le minot de blé, au prix du marché, vaut alors 4 livres et ne cessera d'augmenter. Le fermier, François Séguin, est un menuisier-charpentier bien établi de Terrebonne qui se lance dans le commerce des grains à la faveur d'un marché très favorable aux entrepreneurs et aux marchands. La guerre d'Indépendance des Treize Colonies va bien sûr bouleverser les échanges et entraîner l'arrêt du commerce extérieur. Mais le ravitaillement des armées, très avantageux, l'accaparement des stocks et la spéculation aidant, prendra le relais. Toutefois cette nouvelle conjoncture apparait comme s'achève la période couverte par notre étude.

La performance de l'entreprise de scierie est plus difficile à évaluer. Mises à part celles livrées à l'intendant par Lepage, les pièces de bois fabriquées à Terrebonne sont entrées dans les circuits d'échanges en laissant peu d'indications sur leur destination. Il est vrai que le commerce du bois au XVIII ${ }^{e}$ siècle, plus particulièrement à l'intérieur, et encore plus sous le Régime français, est un domaine mal connu. Certains indices nous permettent cependant de croire que, à partir du moment où La Corne a acheté la seigneurie de Terrebonne, le marché local a absorbé la plus grande part de la production de ses moulins à scie, du moins jusqu'à ce que Jacques Perras en devienne sociétaire et gestionnaire en $1757^{80}$. Le fait que La Corne se soit associé à Charles Gervaise en 1745 pour l'exploitation de ses deux moulins à scie milite en faveur de cette thèse ${ }^{81}$, car Gervaise est membre d'une famille de marchands de bois engagés dans ce commerce à l'échelle locale et régionale depuis un bon moment ${ }^{82}$.

78. Cinquante mille minots de blé y sont transformés cette année-là. Fernand Ouellet, Le Bas-Canada, 1791-1840 (Ottawa, 1980), 18, cité par Claude Pronovost, L'économie marchande au Bas-Canada: le bourg de Terrebonne dans la première moitié du XIXe siècle, mémoire de M.A. (Histoire), Université de Montréal, 1988, 16.

79. Bail à ferme et loyer du moulin de Terrebonne à François Séguin, notaire Jacques Dufault, 10 mars 1775 .

80. À ce moment-là, il n'y a plus qu'un seul moulin, à deux scies.

81. Société entre Louis de La Corne et Charles Gervaise, notaire Louis-Claude Danré de Blanzy, 2 février 1745; bail à loyer d'un moulin à scie entre les mêmes parties (ce bail n'a de bail que le nom: il présente toutes les caractéristiques d'un contrat de société), notaire François Simonnet, 15 septembre 1745.

82. Nicolas Gervaise est fermier des moulins de Berthier et a été dans le passé associé à un Louis Gervaise dans des marchés de bois. Charles assumera à son tour la gestion du moulin à scie de Berthier en 1754, au terme de son association avec La Corne. Société entre monsieur de Lestage et Nicolas Gervaise pour l'exploitation des moulins à farine et à scie de Berthier, notaire Jean-Baptiste Adhémar, 13 septembre 1739; vente de coupe de bois par Jean-Baptiste Varin à Nicolas et Louis Gervaise, notaire Jean-Baptiste Adhémar, 26 avril 1734; engagement de Joseph Bourgeois, pour effectuer divers travaux au moulin à scie, par Charles Gervaise, marchand de bois de sciage de Berthier, notaire Georges Hodiesne, 20 octobre 1754. 
Il s'agit d'ailleurs du secteur le plus actif de l'industrie du bois. On ne sait pas si les exportations en France ont repris pendant l'entredeux-guerres - pour ce qui est des Antilles les historiens se contredisent ${ }^{83}$ - mais, en 1756, Bigot décrète l'embargo sur les sorties de bois de la colonie. Entre-temps, le commerce intérieur a pris de l'expansion - nous parlons ici des transactions entre particuliers - avec l'augmentation de la population et la croissance des villes. Le relevé des marchés de fourniture de bois de construction et de bois rond transigés devant notaire entre 1701 et 1765 montre une montée lente mais continue des échanges jusqu'aux années 1740 et une croissance marquée au cours des décennies suivantes: tout près de $60 \%$ de toutes les transactions s'appliquent, en effet, à la période $1750-1765^{84}$.

$\mathrm{Au}$ moment où il acquiert la seigneurie de Terrebonne, La Corne bénéficie donc d'un marché intérieur de plus en plus étendu et profitable. Autant la demande de l'intendance (ce sont, en effet, tous les secteurs de la production et du commerce qui sont mis à contribution par la hausse de la demande gouvernementale ${ }^{85}$ ) que celle des particuliers croissent considérablement au cours des années de son administration. Et La Corne joue sur les deux plans, s'associant successivement à un marchand de bois bien intégré aux circuits d'échanges locaux et au commis de Joseph Cadet ${ }^{86}$, Jacques Perras qui, on le suppose, écoule la production du moulin à scie de Terrebonne dans les circuits de distribution des fournitures militaires.

En 1765, madame de Lacorne afferme son moulin à scie à Alexandre Dumas. Il n'est désormais plus question de société à profits et risques partagés et, bien entendu, elle ne fournit plus la matière première. Plus question non plus pour la seigneuresse, comme au temps de son association avec Charles Gervaise, de prendre sur elle la responsabilité de la vente d'une partie du bois scié à Terrebonne ${ }^{87}$. Elle

83. D'après le tableau des exportations de Jean A. Lunn, les sorties vers les îles du Vent auraient cessé dès 1739 . Les tableaux de Jacques Mathieu sur le commerce des planches, madriers et bordages avec les Antilles vont dans le même sens. Alana G. Reid, pour sa part, prétend que les échanges (tous produits confondus) n'ont jamais repris sinon qu'à un rythme très irrégulier entre les deux colonies durant l'entre-deux-guerres. Enfin, Louise Dechêne réfère aux travaux de James Pritchard qui soutient que «le commerce Québec-Antilles entre 1749 et 1755 porte essentiellement sur le bois et les poissons", sans toutefois donner d'indication sur le volume et le rythme des échanges en question. Jean A. Lunn, Développement économique..., 315-317; Jacques Mathieu, Le commerce..., 244 et 251-252; Alana G. Reid, «Intercolonial Trade During the French Regime», Canadian Historical Review, 32,3 (septembre 1951): 249-250; Louise Dechêne, Le partage..., 111.

84. Solange De Blois, Possibilités et limites..., 148.

85. Louise Dechêne, Le partage..., 144.

86. Précisons qu'ici Perras agit pour son propre compte et non, comme pour la minoterie, en tant qu'agent de Cadet.

87. À l'époque de Gervaise, c'est elle qui gérait l'entreprise seigneuriale durant les fréquentes absences de son mari. 
peut dorénavant retirer les revenus de sa scierie (comme c'est le cas depuis longtemps pour la minoterie) sans avoir à participer ni à la gestion de l'exploitation ni à la commercialisation des produits. Manifestement, l'industrie du bois est de plus en plus lucrative. De toute évidence aussi, les Lacorne ont, tout au long de leur administration et contrairement à l'abbé Lepage, retiré de bons revenus de leur scierie ${ }^{88}$, la place qu'ils occupaient (sous le Régime français) dans les réseaux économiques et politiques de la colonie jouant, tout comme la conjoncture (sous les deux Régimes), indiscutablement en leur faveur.

\section{CONCLUSION}

L'impatience est mauvaise conseillère en affaire: Louis Lepage a sauté une étape cruciale dans sa progression vers le haut, celle de la constitution d'un capital, d'une assise financière sur laquelle appuyer ses entreprises ${ }^{89}$, et s'est retrouvé Gros-Jean comme devant à vingtcinq ans de distance.

Un pied dans la réalité et l'autre dans un avenir meilleur, il n'a cessé d'investir de l'argent emprunté en misant sur un développement économique qu'il croyait imminent, mais qui se révélait chaque fois illusoire. Il aurait fallu, pour compenser la faiblesse fondamentale de son entreprise, qu'explosent les structures de l'économie du temps, que la colonie abrite une population plus dense, que se modifient les conditions de l'offre et de la demande dans tous les secteurs. Peut-être Lepage a-t-il escompté de tels changements.

Cependant, il nous faut distinguer, dans l'analyse de l'échec de Lepage en affaires, entre la minoterie d'une part, et la scierie et les autres entreprises, d'autre part - soulignons qu'il a produit du brai et du goudron à une époque, en plus d'avoir voulu implanter une forge.

La meunerie, on l'a vu, était bien intégrée à l'économie globale et rapportait, en année normale, de substantiels bénéfices à son propriétaire quoique ne suffisant pas à couvrir son déficit accumulé. Par contre le bois de sciage, tout comme le brai et le goudron qui sortaient

88. De toute l'entreprise seigneuriale, devrions-nous dire, comme les propos d'un contemporain en témoignent. En 1752, l'ingénieur Louis Franquet estimait à 12000 livres les revenus des terres et établissements de Terrebonne. Or les rentes ne rapportaient pas plus de 1417 livres cette année-là, et les lods et ventes 43 livres 10 sols, soit un total de 1460 livres 10 sols. Les moulins et le domaine de la Pointe-au-Pin, s'il existait toujours, rapportaient donc un bénéfice brut d'environ 10500 livres, ce qui est appréciable.

89. De plus, il ne possédait aucun bien foncier avant l'achat de Terrebonne mise à part une petite portion de Rimouski qu'il a cédée à l'héritier principal en échange d'une rente de 200 livres par an, sa vie durant. Notaire Jacques Pinguet de Vaucour, 10 octobre 1735. Précisons que le commerce, la voie habituelle pour constituer un capital, est une activité incompatible avec l'état ecclésiastique: il est interdit par le droit canon. 
de Terrebonne et une bonne part de la production des forges projetées, étaient destinés aux chantiers navals de Rochefort. En d'autres mots, l'entreprise de meunerie à part, Lepage n'avait qu'un seul client: le roi, et c'est là où le bât blesse.

Bien sûr, l'État paie comptant, lorsqu'il paie, et c'est sans doute un des motifs qui ont poussé Lepage, toujours aux abois, à se jeter dans les bras du prince. Mais aussi il a vu trop grand, présumant des possibilités de production de sa scierie. Et de toute façon, les marchés avec le roi, pour des produits destinés à la France, ont tendance à se resserrer bien plus qu'à prendre de l'expansion, suivant l'état des finances et des besoins de la Marine et la place qu'occupe l'industrie coloniale canadienne dans l'échelle des priorités des dirigeants métropolitains qui, ne l'oublions pas, font face à une multitude de quémandeurs. Ce sont, en effet, des motifs d'ordre financier et stratégique (qui doivent aussi prendre en compte les nécessités du système de privilège de l'Ancien régime), bien plus que l'application bornée de certains principes mercantilistes, comme on l'a souvent prétendu dans l'historiographie, qui poussent le ministre à refuser son aval au projet d'implantation d'une forge à Terrebonne, tout comme autrefois il avait mis le holà à celui d'ouverture d'un chantier naval royal à Québec.

Mais si Lepage recherchait la clientèle du roi, c'est aussi parce qu'il n'avait pas d'autre choix étant donné sa situation financière ${ }^{90}$. Toujours à sec, il se trouvait en plus sans alliance utile d'aucune sorte - familiale, commerciale ou politique - et, en conséquence, n'était associé à aucun des réseaux économiques de la colonie. Ses liens avec certains officiers civils et des marchands sont ceux d'un débiteur visà-vis de ses créanciers et n'ont rien d'un partenariat d'une quelconque nature. Même son association avec les cousins d'Ailleboust est établie sur une base inégalitaire puisque ce sont eux qui fournissent la totalité du capital non fixe.

Le cas Lepage montre que, au moins en tant de paix, les contrats de fourniture à l'État ne sont pas toujours avantageux et que, si la conjoncture a pu le desservir, sa stratégie face aux possibilités offertes par les marchés découlait davantage de la précarité de sa situation financière et de son isolement en affaire que d'un calcul pur et simple. Dans ces conditions, il aurait été surprenant, à une époque où les jeux

90. L'intendant se montrait disposé, afin qu'il puisse faire face aux frais d'opération qui ne souffrent pas de délai (salaires, etc.), et c'est là un avantage séduisant pour un entrepreneur dépourvu de capital, à le payer à l'avance pour du bois encore à livrer. Lepage n'a jamais réussi à rembourser la totalité des avances allouées. Hocquart au ministre, 25 octobre 1730, AC, C11 A, volume 53, ff. 181-182; idem, 30 septembre 1731, AC, C11A, 20 septembre 1731, volume 55, f. 28. «Etat de ce que moy Louis Lepage de Sainte Claire prestre doit, annexé au contrat de vente de la seigneurie de Terrebonne», notaire L.-C. Danré de Blanzy, 15 janvier 1745. 
d'alliances sont d'une si grande importance pour quiconque cherche à accéder aux sommets, qu'il réussisse à s'enrichir.

Louis de La Corne bénéficiait de son côté de tout ce qui a fait défaut à son prédécesseur. Fortune mobilière acquise dans le commerce des fourrures, assise foncière par son mariage avec Élisabeth de Ramezay, statut social élevé, solides alliances familiales, commerciales et politiques, conjoncture favorable; tous ces avantages ont permis au couple La Corne-Ramezay de consolider sa position et d'entrer de plain-pied dans des réseaux d'échanges fort utiles. Mais rendons à César ce qui est à César et n'oublions pas que la qualité et la puissance des moulins de Lacorne sont pour beaucoup dans sa réussite. Incontestablement, l'intérêt du munitionnaire du roi envers Terrebonne, qu'il transforme ni plus ni moins en centre de production et de distribution de farines pendant toute la durée de la guerre de Sept Ans, s'explique avant tout par le fait que cette minoterie est fiable et avantageusement située sur la route des pays d'en haut et sur l'axe de navigation vers Québec ${ }^{91}$.

Il est bien évident que la conjoncture a généralement servi les La Corne dans la mesure, bien sûr, où ils se trouvaient bien placés pour en profiter. D'ailleurs, les modes d'exploitation des moulins qu'ils ont adoptés tout au long de leur administration reflètent la congruence de l'entreprise et des marchés. L'activité aux moulins a crû sans arrêt à partir de la guerre de Succession d'Autriche, attirant dans sa périphérie un grand nombre de gens cherchant à s'enrichir dans le ravitaillement militaire. Ce sont des années fastes pour les propriétaires et le village de Terrebonne qui prend alors son élan et verra bientôt sa croissance s'accélérer.

Entre 1763 et 1775 , huit nouveaux marchands dont trois au moins sont engagés dans le commerce des grains et farines, s'installeront à Terrebonne pour de plus ou moins longues périodes ${ }^{22}$. Voilà le signe certain que les moulins (particulièrement celui à farine) génèrent une activité soutenue au bourg. La présence d'un seigneur entreprenant est sans aucun doute un facteur essentiel de l'émergence de l'industrie dans le cadre seigneurial, mais une fois celle-ci solidement implantée,

91. On retrouve à Berthier le même ensemble de facteurs qu'à Terrebonne et on est en droit de supposer que Cadet a fait de la seigneurie des Lestage une autre des têtes de pont de son système de distribution car, à la fin de l'été 1759, 200 soldats partent de Québec pour aller faire les récoltes à Terrebonne et à Berthier. Nicolas Renaud D'Avesne de Meloizes, «Journal militaire de l'année 1759, vendredi 7 septembre 1759», Rapport de l'archiviste de la province de Québec (1928-1929): 72.

92. Au total entre 1728 et 1775,17 marchands séjourneront à Terrebonne dont 8 sont des marchands de grains. Voir la «Liste des marchands domiciliés à Terrebonne 1728-1775», dans Solange De Blois, Possibilités et limites..., 102. 
le rôle du seigneur perd de l'importance et alors, le plus souvent, et c'est le cas des Lacorne au cours des dernières décennies de notre période, il cesse de se mêler directement de gestion pour devenir rentier. 